\title{
Identificação da artéria de Adamkiewicz usando angiotomografia computadorizada por multidetectores (ATCM)*
}

\section{Identification of the Artery of Adamkiewicz Using Multidetector Computed Tomography Angiography (MCTA)}

\author{
Flávio P.F. Piola1® Marcello H. Nogueira-Barbosa ${ }^{2}$ \\ Maurício F. Barbosa ${ }^{4}$ Carlos F.P. Silva Herrero ${ }^{1}$ \\ ${ }^{1}$ Departamento de Biomecânica, Medicina e Reabilitação do Aparelho \\ Locomotor, Faculdade de Medicina de Ribeirão Preto, Universidade \\ de São Paulo, Ribeirão Preto, SP, Brasil \\ 2 Divisão de Radiologia, Faculdade de Medicina de Ribeirão Preto, \\ Universidade de São Paulo, Ribeirão Preto, SP, Brasil \\ 3 Instituto de Radiologia Presidente Prudente, Presidente Prudente, \\ SP, Brasil \\ ${ }^{4}$ Faculdade de Medicina de Botucatu, Universidade Estadual de São \\ Paulo, Botucatu, SP, Brasil
}

Rev Bras Ortop 2020;55(1):70-74.

\author{
Daniel A.C. Maranho ${ }^{1}$ Adélson A. Martins ${ }^{3}$
}

Endereço para correspondência Flávio P.F. Piola, PhD, Departamento de Biomecânica, Medicina e Reabilitação do Aparelho Locomotor, Faculdade de Medicina de Ribeirão Preto, Universidade de São Paulo, Avenida dos Bandeirantes, 3900, Ribeirão Preto, SP, 14048-900, Brasil (e-mail: flaviopfpiola@gmail.com).

\section{Resumo \\ Palavras-chave \\ - angiografia/métodos \\ - artéria/patologia \\ - tomografia computadorizada multidetectores \\ - paraplegia \\ - medula espinal}

Objetivo Avaliar a artéria de Adamkiewicz por angiotomografia computadorizada por multidetectores (ATCM) em uma população brasileira.

Métodos Dois observadores independentes avaliaram 86 exames de ACTM. As variáveis estudadas incluíram a identificação da artéria de Adamkiewicz no nível de origem e o lado de entrada da artéria na coluna vertebral.

Resultados A artéria de Adamkiewicz foi identificada em 71 (82,5\%) exames. O nível de origem foi identificado entre a 9a e a 11a vértebras torácicas (T9 e T11) em 56 (79,2\%) pacientes. Em 65 (91,5\%) pacientes, a artéria foi identificada no lado esquerdo. A identificação da artéria de Adamkiewicz usando ACTM mostrou elevada reprodutibilidade.

Conclusões Obtivemos resultados consistentes com os da literatura prévia quanto à identificação da artéria de Adamkiewicz utilizando angiotomografia computadorizada por multidetectores. Nossos resultados sugerem que a ATCM pode ser considerada como uma opção para identificar a artéria de Adamkiewicz. Além disso, encontramos uma distribuição da artéria de Adamkiewicz na população brasileira semelhante à de outras populações, com a artéria de Adamkiewicz originando-se mais comumente no lado esquerdo, entre a 8a e a 12a vértebras torácicas (T8-T12).
Trabalho desenvolvido no Departamento de Biomecânica, Medicina e Reabilitação do Aparelho Locomotor, Faculdade de Medicina de Ribeirão Preto, Universidade de São Paulo, Ribeirão Preto, SP, Brasil. recebido

31 de Agosto de 2018 aceito

16 de Outubro de 2018
DOI https://doi.org/

10.1055/s-0039-1700829. ISSN $0102-3616$.
Copyright $(2020$ by Sociedade Brasileira License terms de Ortopedia e Traumatologia. Published by Thieme Revinter Publicações Ltda, Rio de Janeiro, Brazil 


Abstract
Keywords
- angiography/methods
- arteries/pathology
- multidetector
computed
tomography
- paraplegia
- spinal cord

Objective The aim of the present study is to evaluate the artery of Adamkiewicz by multidetector computed tomography angiography (MCTA) in a Brazilian population. Methods Two independent observers evaluated 86 coronaryMCTA examinations. The variables studied included the identification of the artery of Adamkiewicz at its origin level, and its entry side in the spine.

Results The artery of Adamkiewicz was identified in 71 (82.5\%) examinations. The origin level was identified between the 9th and 11th thoracic vertebrae (T9 and T11) in $56(79.2 \%)$ patients. In $65(91.5 \%)$ patients, the artery was on the left side. The identification of the artery of Adamkiewicz using MCTA showed high reproducibility. Conclusions Our results were consistent with the literature regarding the identification of the artery of Adamkiewicz using MCTA, suggesting that this technique should be considered as an option to identify this structure. In addition, we found that the distribution of the artery of Adamkiewicz in the Brazilian population similar to that of other populations, that is, its most common origin is at the left side, between the 8th and 12th thoracic vertebrae (T8-T12).

\section{Introdução}

O déficit neurológico no pós-operatório de procedimentos cirúrgicos vasculares e espinhais é uma grave complicação potencial devido ao envolvimento e comprometimento do suprimento sanguíneo da coluna vertebral. ${ }^{1}$ Estudos anteriores mostraram uma incidência de paraplegia e paraparesia pós-operatória de $11,3 \% .^{2}$ Puertas et al. ${ }^{3}$ relataram uma incidência de lesão neurológica permanente de $4 \%$ nos pacientes tratados cirurgicamente para a correção da escoliose idiopática através de instrumentação e artrodese por via posterior. A artéria de Adamkiewicz (AAK) fornece o principal suprimento sanguíneo para a medula espinhal, do nível T8 para o cone medular. ${ }^{4-6}$ Portanto, a identificação pré-operatória da AAK desempenha um papel crítico na prevenção da isquemia ou infarto da medula espinhal.

A taxa de identificação da AAK pela angiografia espinhal seletiva varia entre 65 e $86 \%^{1,7}$ No entanto, a angiografia seletiva da coluna pode ser demorada e tecnicamente desafiadora, além de apresentar riscos potenciais para o paciente. Complicações relacionadas ao procedimento foram relatadas em $1,2 \%$ dos pacientes. ${ }^{8}$ A angiotomografia computadorizada por multidetectores (ATCM) é um método alternativo não invasivo e eficiente que permite a visualização da $A A K .{ }^{8-10}$

A identificação da AAK é viável com as técnicas atuais da ATCM. $^{8,10-12}$ No entanto, esses métodos frequentemente exigem não apenas sequências ou protocolos específicos, mas também estações de trabalho caras e elaboradas, e podem consumir recursos. ${ }^{11}$ Assim, na maioria dos centros, a identificação de AAK não faz parte da avaliação de rotina pré-operatória nas cirurgias de coluna vertebral e vasculares.

Embora as demonstrações da AAK tenham sido bem documentadas na Europa, na América do Norte, e na Ásia, a visualização da AAK pode variar entre diferentes regiões, e poucos estudos em populações latino-americanas detalharam a localização da AAK. ${ }^{8,11-14}$ Nossa hipótese era que a posição da AAK diferiria dos dados previamente relatados. $O$ objetivo do presente estudo foi avaliar a localização da AAK em uma amostra populacional brasileira.

\section{Materiais e Métodos}

O Comitê de Ética em Pesquisa do Hospital das Clínicas da Faculdade de Medicina de Ribeirão Preto da Universidade de São Paulo (Parecer $n^{\circ}$ 957.443, data da Relatoria 13/11/2014) aprovou o presente estudo. $O$ estudo é de caráter retrospectivo e foi composto por avaliações consecutivas de ATCM de 96 pacientes que foram submetidos a investigações de doença arterial coronariana de janeiro de 2015 a janeiro de 2016 . Indivíduos com evidência de patologia aguda ou pacientes com sinais de cirurgia prévia na coluna foram excluídos do estudo. Um total de 86 pacientes preencheram os critérios de inclusão e foram considerados para nossa avaliação. Avaliamos as imagens de ATCM de 57 homens e 29 mulheres. A idade média dos pacientes do sexo masculino foi de 56,9 anos (variação de 31-82 anos) e a idade média das pacientes do sexo feminino foi de 59,7 anos (variação de 18-83 anos).

\section{Aquisição e Reconstrução de Imagens}

Todos os dados das ATCM foram adquiridos usando o scanner de tomografia computadorizada Aquilion Prime 160 cortes por rotação (Toshiba Medical Systems Corporation, Otawarashi, Tochigi, Japão). Os parâmetros de escaneamento foram 120 kV, 250 mA em média, 0,50 de colimação, e um período de rotação de 0,35 segundos (pitch 0,641). Reconstruções multiplanares de tomografia computadorizada (TC) foram obtidas no plano axial com $0,5 \mathrm{~mm}$ de espessura, intervalo de reconstrução de $0,3 \mathrm{~mm}$, e campo de visão de 300 a $340 \mathrm{~mm}$. Além disso, reconstruções multiplanares coronais oblíquas de $8 \mathrm{~mm}$ de espessura usando a técnica de máxima intensidade de projeção (MIP) foram obtidas usando uma estação de trabalho Vitrea (Vital Images, Inc., Minnetonka, MN, EUA).

\section{Protocolo de Injeção Intravenosa e de Escaneamento}

$O$ protocolo de injeção envolveu o uso do agente de contraste não-iônico Iopamidol, 755 mgI / m (Iopamiron 370, Patheon, Ferentino, Frosinone, Itália). Uniformemente, $80 \mathrm{~mL}$ do contraste foram administrados através de uma linha IV de 16 a 18 gauge colocada na veia antecubital a uma taxa de fluxo de 


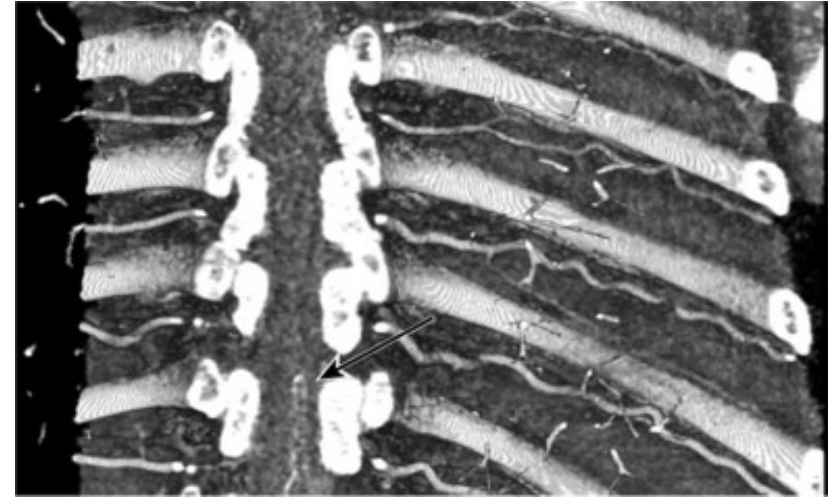

Fig. 1 Configuração típica da AAK em aspecto de grampo de cabelo.

$5 \mathrm{~mL} / \mathrm{s}$, seguido por um bolus de $40 \mathrm{~mL}$ de solução salina. O escaneamento foi iniciado 7 segundos após o contraste ter atingido 180 unidades Hounsfield (UH) na região de interesse (RDI), posicionadas na aorta ascendente.

\section{Análise de Imagem}

O segmento distal da AAK e da artéria espinhal anterior tipicamente demonstra uma configuração em grampo de cabelo (-Figura 1). Portanto, para identificar a AAK, aplicamos os seguintes critérios: uma estrutura vascular contínua com um curso reto que se estende de uma artéria intercostal ou lombar, subindo na superfície média sagital anterior da medula espinhal, com a curva característica em grampo de cabelo na anastomose com a artéria anterior da coluna. Inicialmente, reconstruções multiplanares coronais oblíquas obtidas por meio da técnica MIP foram utilizadas para identificar o giro na junção da artéria espinhal anterior e da grande artéria radiculomedular anterior. O próximo passo foi identificar o nível de origem e o lado em que a AAK estava localizada (- Figura 2).

Reprodutibilidade Interobservador e Intraobservador Dois observadores avaliaram independentemente os exames tomográficos usando a mesma estação de trabalho (Vitrea, Vital Images, Inc.). O observador 1 foi um radiologista com 15 anos de experiência na realização e interpretação de angiografias diagnósticas, e o observador 2 foi um cirurgião de coluna com 20 anos de prática. O observador 1 realizou uma avaliação aleatória

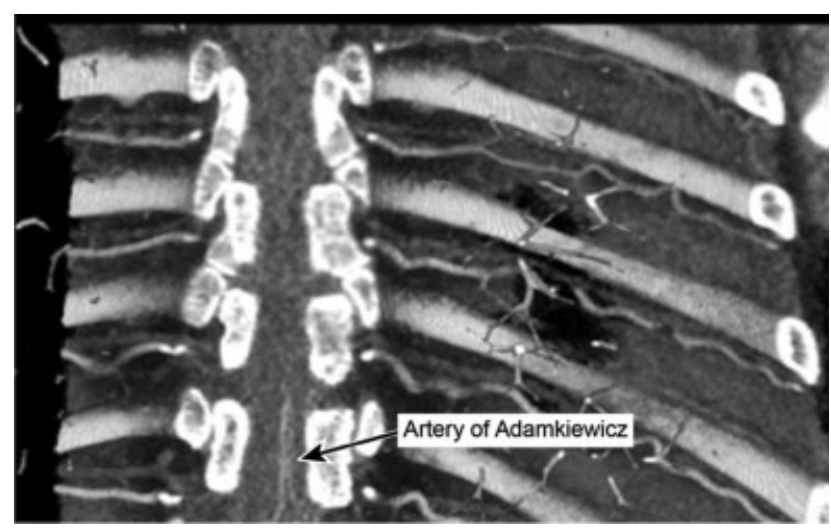

Fig. 2 Entrada da AAK pelo lado esquerdo ao nível de T11. adicional com um intervalo de 2 meses entre as avaliações para a estimativa da confiabilidade intraobservador.

\section{Análise Estatística}

Avaliamos a reprodutibilidade da identificação da AAK calculando as concordâncias interobservador e intraobservador da detecção de AAK, o nível de origem e o lado da localização usando o valor kappa de Cohen. Considerou-se que os valores apresentaram concordância fraca entre 0 e 0,20 , concordância pequena entre 0,21 e 0,40 , concordância moderada entre 0,41 e 0,60 , concordância substancial ou boa quando estavam entre 0,61 e 0,80 e concordância quase perfeita ou muito boa quando estavam entre 0,81 e 1,0. Usamos o software Stata13 (StataCorp, 2013, College Station, TX, EUA para a análise estatística.

\section{Resultados}

O observador 1 identificou a AAK em 71 pacientes (82,5\%) durante a primeira análise de imagem. Assim, em 15 pacientes $(17,5 \%)$ da amostra do estudo, o observador 1 não detectou a AAK. Em relação ao lado da localização, quando identificado, a AAK originou-se do lado esquerdo do corpo em 65 pacientes (91,5\%) e do lado direito em 6 pacientes (8,5\%). A nível segmentar variou do $6^{\circ}$ nível torácico ao $1^{\circ}$ espaço intervertebral lombar. Os níveis de origem mais frequentes em nossa amostra foram T10, T9, e T11 em 23 (32,5\%), 19 (26,7\%) e 14 (20\%) exames, respectivamente. Por outro lado, os níveis de origem menos comuns incluíram os níveis de T6 e L1 (-Tabela 1). Não encontramos anomalias da AAK ou duplicações ipsilaterais ou bilaterais em nosso estudo. Os resultados relacionados ao lado e ao nível de origem da AAK estão demonstrados na - Tabela 1. Após uma seleção aleatória de 18 exames (20\% da amostra), o observador 1 realizou a segunda avaliação. A AAK foi identificada em 17 exames (95\%), e em 1 exame (5\%), a AAK não foi identificada. O nível de origem variou de T6 a L1, e a AAK originou-se do lado esquerdo em 16 pacientes (94\%) e do lado direito em apenas 1 paciente (6\%). A concordância intraobservador foi perfeita $($ kappa $=1)$ não só para a identificação da AAK, mas também

Tabela 1 Nível de origem e lado de entrada da AAK em 71 pacientes

\begin{tabular}{|l|l|l|}
\hline Nível de origem da AAK & \multicolumn{2}{|l|}{ Lado da AAK } \\
\hline & Esquerdo & Direito \\
\hline T5 & 0 & 0 \\
\hline T6 & 1 & 0 \\
\hline T7 & 0 & 0 \\
\hline T8 & 5 & 0 \\
\hline T9 & 17 & 2 \\
\hline T10 & 22 & 1 \\
\hline T11 & 14 & 3 \\
\hline T12 & 5 & 0 \\
\hline L1 & 1 & 0 \\
\hline
\end{tabular}

Abreviaturas: AAK, artéria de Adamkiewicz; L, lombar; T, torácico. 
para o lado e nível de origem. O observador 2 avaliou a AAK nos mesmos 17 (95\%) exames e não foi capaz de identificar a AAK em 1 exame. Dos 17 exames, o nível de origem variou de T6 a L1 e a AAK originou-se do lado esquerdo em 16 pacientes (94\%) e do lado direito em 1 paciente (6\%). A concordância interobservador também foi perfeita (kappa $=1$ ) para a demonstração da AAK e a determinação do lado e do nível de origem.

\section{Discussão}

Nossos resultados revelaram que a ATCM é uma técnica viável para identificar a artéria de Adamkiewicz (AAK), e nossos resultados são consistentes com os dados previamente relatados. . $^{8,11-14}$

Partindo do princípio de que a anatomia da AAK em nossa população pode diferir de outras populações, o estudo anatômico da localização dessa artéria torna-se importante e tem implicações terapêuticas potenciais, especialmente para o planejamento de procedimentos que poderiam comprometer a AAK por subsequente isquemia de a medula espinhal distal. ${ }^{15}$ Como muitas outras variações anatômicas são encontradas em raças e etnias distintas, consideramos que os dados obtidos a partir de estudos anteriores devem ser interpretados com cautela, pois podem não se aplicar universalmente. ${ }^{15-17}$

Embora vários estudos de imagem tenham abordado o reconhecimento da $A A K$, ainda existe um debate sobre a melhor técnica para identificar a AAK. ${ }^{17}$ Vários estudos descreveram complicações relacionadas ao procedimento quando se utilizou angiografia seletiva da coluna vertebral. ${ }^{1,7}$ Publicações mais recentes têm favorecido técnicas menos invasivas, como a angiorressonância magnética (ARM) ou a angiotomografia computadorizada (ATC). ${ }^{10}$ No entanto, como esses métodos podem exigir protocolos especiais, administração de contraste intravenoso, tempo, e recursos, na maioria dos centros, a identificação de AAK não faz parte da análise clínica de rotina. A ATC, embora não seja invasiva, expõe o paciente a uma dose significativa de radiação ionizante. Decidimos avaliar a localização da AAK através do ATCM, um exame bem conhecido que é empregado para a vascularização coronariana.

Estudos anteriores da ATCM sobre a visualização da AAKe suas características anatômicas incluíram populações européias, americanas, e asiáticas. ${ }^{8,12-14}$ Apesar da aumento de procedimentos vasculares e espinhais com implicações na alteração na perfusão da medula espinhal, podem existir características anatômicas distintas no tratamento de diferentes grupos étnicos. Melissano et al. ${ }^{18}$ revisaram dados publicados sobre a visualização da AAK por meio de ARM ou ATC em toda a literatura inglesa e identificaram uma tendência a um resultado homogêneo, independentemente da técnica. O reconhecimento da AAK foi obtido em 466 de 555 exames (83,96\%). Em 384 (83,3\%) casos, a AAK originouse de uma artéria intercostal esquerda e, em 77 (16,7\%) casos, originou-se de uma artéria intercostal direita. Nossa avaliação mostrou que o ATCM é efetiva para visualizar a AAK, e a identificação da AAK foi obtida em 71 pacientes (82,5\%). Consistentemente com estudos anteriores, descobrimos que a AAK se originou do lado esquerdo do corpo em 65 pacientes
(91,5\%) e do lado direito em 6 pacientes (8,5\%). Além disso, as concordâncias intra e interobservador foram perfeitas $($ kappa $=1,0)$ para a avaliação da AAK por meio da ATCM.

Outra possível preocupação, a diferenciação entre a AAKe a veia radiculomedular anterior, é importante, pois a interpretação errônea desses dois vasos pode causar complicações cirúrgicas críticas. ${ }^{18}$ A veia radiculomedular anterior é frequentemente semelhante em forma e tamanho à AAK e pode seguir uma trajetória semelhante à da AAK. Para evitar essa má interpretação, seguimos um protocolo restrito de injeção e escaneamento que foi iniciado $7 \mathrm{~s}$ depois que o agente de contraste atingiu $180 \mathrm{UH}$ na RDI. Assim, concordamos com Utsunomiya et al. ${ }^{19}$ que um protocolo de injeção com uma alta concentração de iodo em um alto fluxo é adequado para identificar a AAK. Além disso, a veia radiculomedular anterior geralmente mostra uma trajetória tortuosa, o que pode ajudar a distinguir a veia da artéria.

Algumas limitações do nosso estudo merecem atenção. Primeiro, esta é uma avaliação retrospectiva. A segunda limitação está relacionada ao fato de que os pacientes foram submetidos a ATCM para a investigação de doença arterial coronariana, e a AAK pode ter sido localizada fora da área estudada. Os níveis mais proximais e distais examinados foram $\mathrm{T} 4 \mathrm{e} \mathrm{L} 1$, respectivamente.

\section{Conclusões}

Obtivemos resultados para a identificação de AAK usando ATCM que foram consistentes com a literatura prévia. Nossos resultados sugerem que a ATCM deve ser considerada uma opção para identificar a AAK. Além disso, encontramos uma distribuição da AAK em pacientes latino-americanos semelhante à de outras populações, com a AAK originando-se mais comumente à esquerda dos níveis T9 a T12.

Conflito de Interesses

Os autores declaram não haver conflito de interesses.

\section{Agradecimentos}

Agradecemos aos funcionários do Instituto de Radiologia de Presidente Prudente por sua assistência com os pacientes.

\section{References}

1 Kieffer E, Fukui S, Chiras J, Koskas F, Bahnini A, Cormier E. Spinal cord arteriography: a safe adjunct before descending thoracic or thoracoabdominal aortic aneurysmectomy. J Vasc Surg 2002;35 (02):262-268

2 Schepens M, Dossche K, Morshuis W, et al. Introduction of adjuncts and their influence on changing results in 402 consecutive thoracoabdominal aortic aneurysm repairs. Eur J Cardiothorac Surg 2004;25(05):701-707

3 Puertas EB, Chagas JC, Oliveira CA, Wajchenberg M, Santos FP, Oliveira VM. Complicações neurológicas no tratamento da escoliose idiopática com instrumentação e artrodese por via posterior. Acta Ortop Bras 1998;6(04):168-172

4 Adamkiewicz A. Die blutgefäße des menschlichen rückenmarkes. I theil, SitzungsBerichte der Kaiserlichen Akademie der Wissenschaften Mathematisch-Naturwissenschaftliche. Classe 1881; 84:469-502 
5 Alleyne CH Jr, Cawley CM, Shengelaia GG, Barrow DL. Microsurgical anatomy of the artery of Adamkiewicz and its segmental artery. J Neurosurg 1998;89(05):791-795

6 Standring S. Gray's anatomy: the anatomical basis of clinical practice. 40th ed. Edinburgh: Churchill Livingstone; 2008

7 Heinemann MK, Brassel F, Herzog T, Dresler C, Becker H, Borst HG. The role of spinal angiography in operations on the thoracic aorta: myth or reality? Ann Thorac Surg 1998;65(02):346-351

8 Takase K, Sawamura Y, Igarashi K, et al. Demonstration of the artery of Adamkiewicz at multi- detector row helical CT. Radiology 2002;223(01):39-45

9 Kudo K, Terae S, Asano T, et al. Anterior spinal artery and artery of Adamkiewicz detected by using multi-detector row CT. AJNR Am J Neuroradiol 2003;24(01):13-17

10 Yoshioka K, Niinuma H, Ohira A, et al. MR angiography and CT angiography of the artery of Adamkiewicz: noninvasive preoperative assessment of thoracoabdominal aortic aneurysm. Radiographics 2003;23(05):1215-1225

11 Nakayama Y, Awai K, Yanaga Y, et al. Optimal contrast medium injection protocols for the depiction of the Adamkiewicz artery using 64-detector CT angiography. Clin Radiol 2008;63(08):880-887

12 Uotani K, Yamada N, Kono AK, et al. Preoperative visualization of the artery of Adamkiewicz by intra-arterial CT angiography. AJNR Am J Neuroradiol 2008;29(02):314-318

13 Fanous AA, Lipinski LJ, Krishna C, et al. The Impact of Preoperative Angiographic Identification of the Artery of Adamkiewicz on
Surgical Decision Making in Patients Undergoing Thoracolumbar Corpectomy. Spine 2015;40(15):1194-1199

14 Nijenhuis RJ, Jacobs MJ, Jaspers K, et al. Comparison of magnetic resonance with computed tomography angiography for preoperative localization of the Adamkiewicz artery in thoracoabdominal aortic aneurysm patients. J Vasc Surg 2007;45(04):677-685

15 Awai K, Hatcho A, Nakayama Y, et al. Simulation of aortic peak enhancement on MDCT using a contrast material flow phantom: feasibility study. AJR Am J Roentgenol 2006;186(02):379-385

16 Fleischmann D, Rubin GD, Bankier AA, Hittmair K. Improved uniformity of aortic enhancement with customized contrast medium injection protocols at CT angiography. Radiology 2000; 214(02):363-371

17 Yoshioka K, Niinuma H, Ehara S, Nakajima T, Nakamura M, Kawazoe K. MR angiography and CT angiography of the artery of Adamkiewicz: state of the art. Radiographics 2006;26(Suppl 1): S63-S73

18 Melissano G, Bertoglio L, Civelli V, et al. Demonstration of the Adamkiewicz artery by multidetector computed tomography angiography analysed with the open-source software OsiriX. Eur J Vasc Endovasc Surg 2009;37(04):395-400

19 Utsunomiya D, Yamashita Y, Okumura S, Urata J. Demonstration of the Adamkiewicz artery in patients with descending or thoracoabdominal aortic aneurysm: optimization of contrastmedium application for 64-detector-row CT angiography. Eur Radiol 2008;18(11):2684-2690 